\title{
LARGE STROKE MINIATURIZED DIELECTRIC ELASTOMER ACTUATORS
}

\author{
S. Rosset, P. Dubois, M. Niklaus, and H.R. Shea ${ }^{*}$ \\ Ecole Polytechnique Fédérale de Lausanne (EPFL), Microsystems for Space Technologies Laboratory, \\ Neuchâtel, Switzerland
}

\begin{abstract}
We report on miniaturized diaphragm dielectric elastomer actuators (DEAs) capable of vertical displacement up to $25 \%$ of their diameter. Low-energy metal ion implantation was used to create patternable and compliant gold electrodes on both sides of suspended 30 $\mu \mathrm{m}$ thick silicone (PDMS) membranes. This technique enables the microfabrication of bucking-mode actuators capable of out-of-plane displacement of up to $500 \mu \mathrm{m}$ for $2 \mathrm{~mm}$ diameter devices at high frequency $(>1 \mathrm{kHz})$. Device speed is limited by the resonant frequency of the device, not by visco-elastic effects or electrical considerations. This represents the largest percentage and fastest displacement of miniaturized DEAs reported to date.
\end{abstract}

\section{KEYWORDS}

Dielectric elastomer actuator; ion implantation; electroactive polymer; compliant electrode

\section{INTRODUCTION}

\section{Dielectric elastomer actuators}

Dielectric elastomer actuators (DEA) are electrically actuated devices consisting of an elastomer sandwiched between two compliant electrodes as shown schematically in Figure 1. When a voltage is applied to the electrodes, an electrostatic pressure $\mathrm{P}$ is generated $P=-\varepsilon_{0} \varepsilon_{r} E^{2}$, where $\varepsilon_{0}$ is the vacuum permittivity, $\varepsilon_{\mathrm{r}}$ is the relative permittivity and $\mathrm{E}$ is the applied electric field. The pressure adds compressive stress to the membrane. Depending on boundary conditions, the compressive stress leads to different actuation modes. We report here on diaphragm type actuators, consisting of an elastomer bonded to a rigid substrate in which circular or square holes have been machined. As shown in Figure 2, once the buckling voltage is exceeded, the membrane deforms and deflects out of the initial plane. The height of the "bump" is controlled by the applied voltage.

Macroscale DEAs combine large strains (over 100\% depending on the configuration [1]) with useful output forces, and have a very high energy density. They are often referred to as artificial muscles, and have been the subject of extensive research since the 1990s. Many functional demonstrators and products based on DEAs have been developed, such as artificial muscles for a robotic arm [2], actuators for hand rehabilitation splints [3], a blimp with biomimetics propulsion [4], energy harvesting devices [5], or manipulators for harsh environments [6].

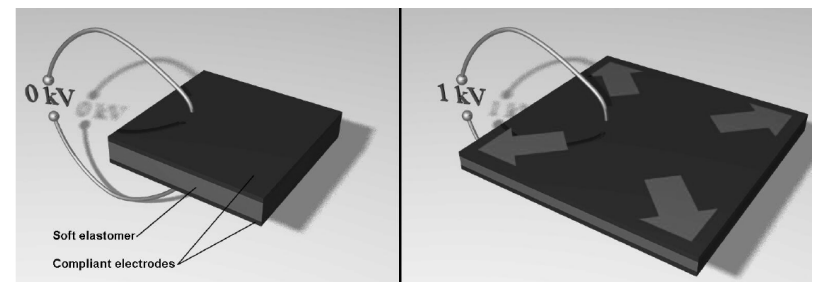

Figure 1: Dielectric Elastomer Actuator principle. Applying a voltage (typically $>1 \mathrm{kV}$ ) to the electrodes creates an electrostatic pressure squeezing the elastomer dielectric [7].

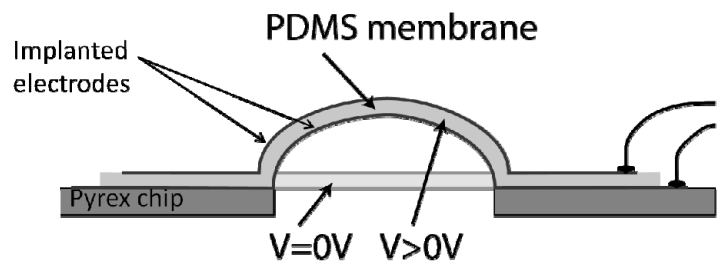

Figure 2: Principle of operation of buckling mode EAP actuator. When the applied electrostatic stress exceeds the buckling threshold, the membrane deforms into a bump, whose height can be controlled by the applied voltage.

Almost all DEA-based applications reported in the literature are macro-scale devices with sizes of a few square centimeters and above. Yet the miniaturization of DEAs is very promising, as such actuators would combine characteristics unmatched by any class of standard MEMS actuator. The electrostatic actuation principle scales well to sub-mm devices. Efforts at miniaturizing DEAs have been mostly hindered by the lack of a microfabricationcompatible technique to make compliant electrodes.

A key requirement on the electrodes used in EAPs (electroactive polymers) is their compliance: for efficient operation, the electrodes must 1) not significantly increase the stiffness of the polymer/electrode stack, and 2) be able to conduct at strains up to $100 \%$. This is far from a trivial requirement because metals have typical Young's modulus 4 to 5 orders of magnitude higher than elastomers, and because sputtered or evaporated metals films cracks and cease to conduct for strains larger than 2 to $3 \%$. Therefore simply depositing a thin metal film on the elastomer will yield devices with neither large deflection nor high efficiency.

For macroscale EAP devices, the solution of choice to make compliant electrodes has been carbon powder, either painted on, mixed with grease, or mixed with the 
unpolymerized elastomer (see for example [1][8][9]). This solution works very well for larger electrodes, but is not well suited to miniaturization, nor is it cleanroom compatible. Danfoss Polypower (Nordborg, Denmark) is commercializing a corrugated silicone membrane onto which a continuous silver film is deposited. This electrode can however not be patterned on the $\mathrm{mm}$ or micron scale, and it allows expansion only in one direction.

Some research groups have patterned metal electrodes on elastomers $[10,11]$, but these electrodes still have an important impact on the stiffness of the polymer membrane.

\section{Ion implanted electrodes}

We introduced low-energy metal ion implantation by filtered cathodic vacuum arc as a successful method to make compliant electrodes for DEAs [12]. We have shown [12][7][15] that low-energy metal ion implantation from an FCVA (filtered cathodic vacuum arc) source allows creating electrodes that have minimal increase in Young's modulus and whose conductivity $(<1 \mathrm{k} \Omega$ /square $)$ is more than sufficient for electrostatic actuation.

Implantation leads to the creation of nanometer-size clusters in the first $50 \mathrm{~nm}$ below the elastomer's surface [15]. The distribution is in part due to the energy variation during the pulsed FCVA implantation. We attribute the ability to conduct at large strain and the small increase in Young's modulus to this microstructure: it appears the clusters are touching yet can slide with respect to each other, thus allowing electrical continuity without significant rigidification.

The Au-implanted electrodes remain conductive up to very large strains. While a thin gold film cracks and hence ceases to conduct at strains between 2 and $3 \%$, Figure 4 shows the resistance vs. strain curves for one $\mathrm{Au}-$ implanted PDMS film. Not only does this sample remain conductive at a strain of $160 \%$, but the electrodes remain conductive even after $10^{5}$ cycles of 0 and $30 \%$ strain [14].

Applying this technology to diaphragm actuators, devices with maximum out-of-plane deflections of $7 \%$ of the membrane's diameter were presented in [12][13]. We present in this paper a new fabrication process that allows achieving out of plane deflections of more than $25 \%$ of the device diameter.

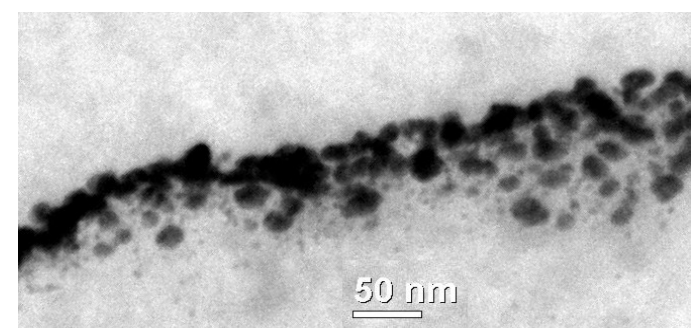

Figure 3: TEM cross-section of an Au-implanted PDMS film implanted using an FCVA source with a $2.5 \mathrm{kV}$ bias. The gold forms clusters at and below the surface of the PDMS. The direction of implantation is from the top of the figure. Details are given in [15].

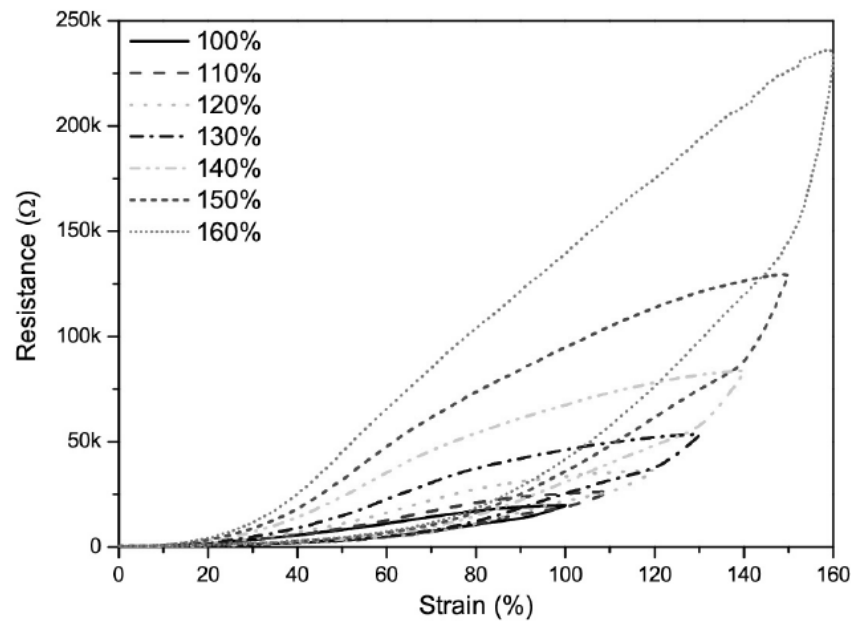

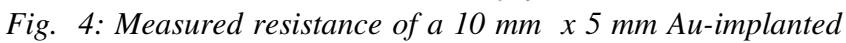
PDMS strip vs. uni-axial strain. The legend displays the maximum strain for each cycle.

\section{ACTUATOR FABRICATION}

The miniaturized EAP actuators consist of a DEA membrane bonded on a Pyrex wafer with circular or square hole of diameter 1 to $3 \mathrm{~mm}$, as shown schematically in figure 5. The devices are fabricated on the chip-scale to keep the total implantation time below 15 minutes.

A $2 \mu \mathrm{m}$ thick photoresist layer is spun on a 4" transfer wafer. The PMDS Sylgard 186 from Dow Corning is prepared by mixing the PDMS with the curing agent with a 10:1 weight ratio. The mixture is diluted with isooctane (PDMS:Solvent 10:9 by weight) to lower its viscosity to allow for spin coating on the photoresist. Final PDMS thickness is 20 to $30 \mu \mathrm{m}$. The PDMS is left to cure for 48 hours, and the wafer is diced into $18 \times 18 \mathrm{~mm}^{2}$ squares. Gold electrodes are sputtered onto the PDMS film, and will serve as contacts to the ion-implanted electrodes, which are deposited through $0.2 \mathrm{~mm}$ thick laser-cut steel shadow- mask.

In parallel Pyrex chips $\left(20 \times 20 \mathrm{~mm}^{2}\right)$ are prepared, onto which gold electrodes are sputtered, and through which holes are laser-cut. The gold electrodes match those on the PDMS. Following an oxygen plasma treatment, the PDMS chip is bonded to the Pyrex chip. No pre-stretch is imposed. The implanted zone on the PDMS thus forms the backside of the actuator. The photoresist is dissolved in acetone, and the transfer wafer is removed. Finally gold electrodes are sputtered on top of the PDMS (i.e., on the side previously in contact with the photoresist) and the second ion implantation is carried out through another shadow mask. Each chip consists of 4 membranes, as shown in Figure 6. 


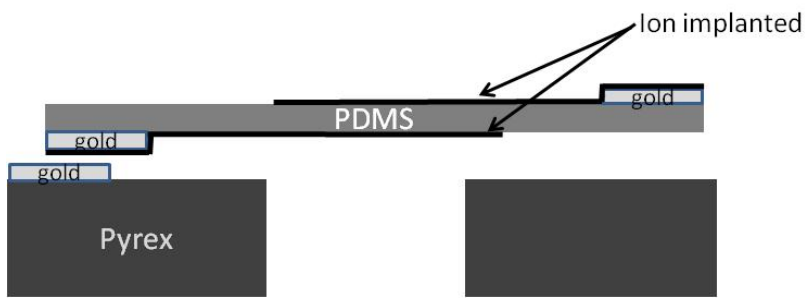

Figure 5: Schematic cross-section of the miniaturized EAP. The doubly-implanted membrane is bonded to the Pyrex chip following an oxygen plasma activation.

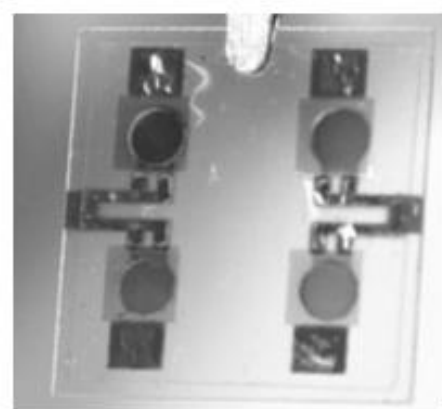

Figure 6: photograph of a $2 \times 2 \mathrm{~cm}^{2}$ chip with 4 actuators.

Our earlier devices were fabricated on conductive silicon chips. This allowed using the silicon wafer as the contact to the bottom implanted layer. Unfortunately, this contact was poor, and led to long (10 to $1000 \mathrm{~ms}$ ) and varying RC time constants, and hence slow device response. This problem was eliminated by patterning gold contacts on the Pyrex chip, ensuring a low resistance contact to the implanted electrodes.

Another advantage of the Pyrex chips is that it allows approximately a doubling of the breakdown voltage compared to the silicon chips. When a contact is applied, the PDMS is locally thinned. This leads to lower breakdown voltage on the conductive silicon chips, but not on the insulating Pyrex chips. Since the Maxwell pressure scales as the square of the voltage, the maximum electrostatic force is roughly four times larger in the device made on Pyrex rather than on silicon.

\section{DEVICE CHARACTERIZATION Static displacement}

Figure 7 is a photograph of a $3 \mathrm{~mm}$ diameter membrane actuated at $1200 \mathrm{~V}$, showing clear buckling behavior. Figure 8 is a plot of the displacement of the center of a membrane vs. DC voltage for three different membrane sizes. The maximum displacement exceeds $25 \%$ of the diameter for the $3 \mathrm{~mm}$ device, approaching the theoretical maximum of $35 \%$ for this geometry due to pullin. The maximum displacement can be increased by using a thinner silicone layer, at the expense of reduced maximum force.

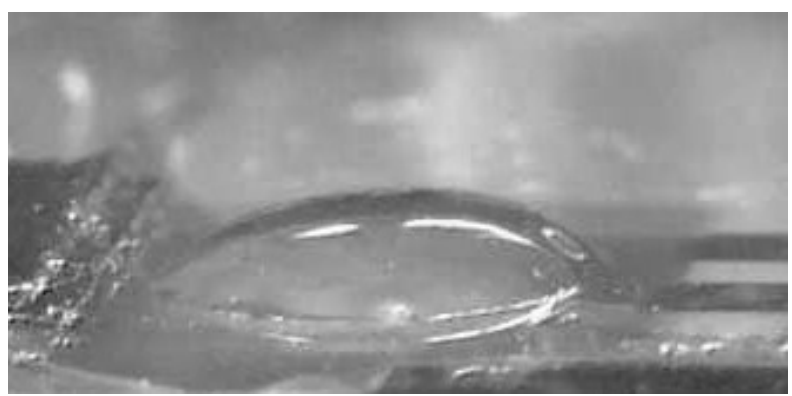

Figure 7: photograph of a $3 \mathrm{~mm}$-diameter EAP actuator with $1200 \mathrm{~V}$ applied between the electrodes.

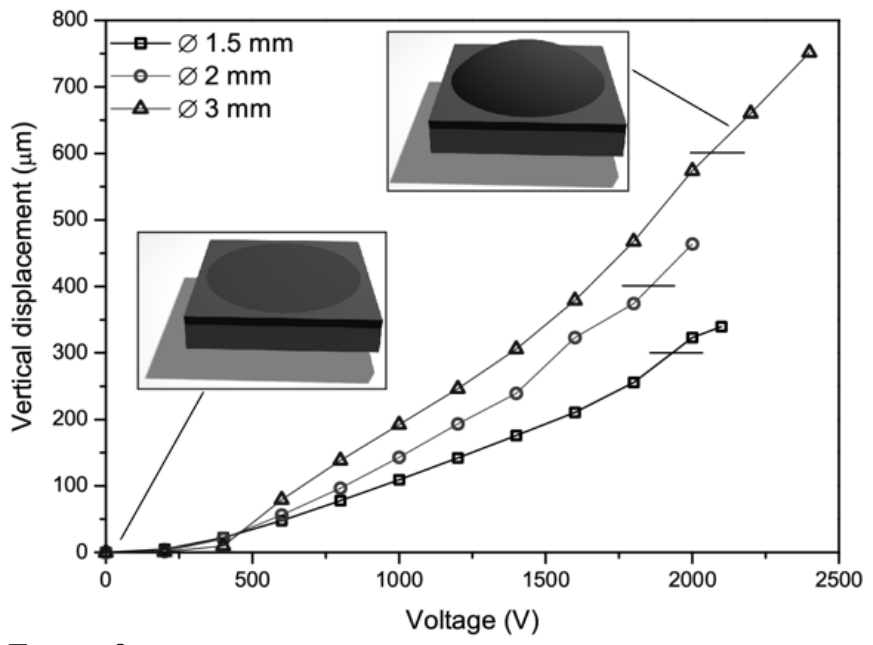

Figure 8: Out-of-plane deflection of the center of the membrane vs. applied voltage for actuators of three different diameters.

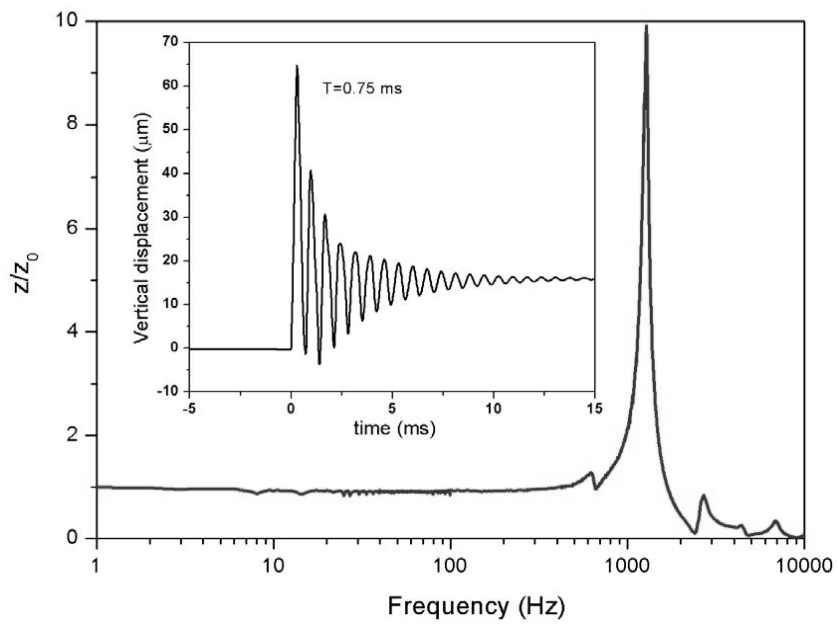

Figure 9: Response of the actuator to an external acoustic pressure, showing resonance at $1.3 \mathrm{kHz}$. Inset: measured deflection in response to a $700 \mathrm{~V}$ voltage step. Mechanically underdamped behavior is seen, corresponding to a $1.35 \mathrm{kHz}$ natural frequency. 


\section{Dynamic response}

The dynamic response of a $3 \mathrm{~mm}$ diameter actuator was measured in response to an acoustic wave and to direct electrical actuation (figure 9). Both actuation methods give a resonance frequency of $1.3 \mathrm{kHz}$, demonstrating the device can be actuated in less than $1 \mathrm{~ms}$. The resonant frequency does not depend on electrical parameters, but only on device geometry and on the Young's modulus and stress of the PDMS film. We reported in [16] a technique for tuning down the resonance frequency by applying an electrostatic stress. This technique is also applicable to the devices bonded on Pyrex substrates.

\section{CONCLUSIONS}

We have used low energy metal ion implantation to fabricate mm-size dielectric elastomer actuators on Pyrex chips, capable of out-of-plane deflection up to $25 \%$ of their diameter and with response time of the order of miliseconds. We have presented a new process flow for the actuators' fabrication, which leads to vertical displacements 4.5 times higher compared to our previous reported results, as well as a much shorter response time. These devices will find uses in arrays of tunable microlenses, beam steering platforms, and micropumps integrated into microfluidic systems.

\section{REFERENCES}

[1] R. Pelrine, R. Kornbluh, Q. Pei, J. Joseph,"High-speed electrically actuated elastomers with strain greater than 100\%", Science 287 (5454), 836-839 (2000).

[2] G. Kovacs, P. Lochmatter, M. Wissler, "An arm wrestling robot driven by dielectric elastomer actuators," Smart Materials and Structures 16(2), s306-s317 (2007).

[3] F. Carpi, A. Mannini, D.D. Rossi, "Elastomeric contractile actuators for hand rehabilitation splints," in Electroactive Polymer Actuators and Devices (EAPAD) 2008, Y. Bar-Cohen, ed., 6927, 692705 (2008).

[4] S. Michel, A. Bormann, C. Jordi, E. Fink, "Feasibility studies for a bionic propulsion system of a blimp based on dielectric elastomers," in Electroactive Polymer Actuators and Devices (EAPAD) 2008, Y. Bar-Cohen, ed., 6927, 69270S (2008).

[5] S. Chiba, M. Waki, R. Kornbluh, R. Pelrine, "Innovative power generators for energy harvesting using electroactive polymer artificial muscles," in Electroactive Polymer Actuators and Devices (EAPAD) 2008, Y. Bar-Cohen, ed., 6927, 692715 (2008).

[6] J. Vogan, A. Wingert, J.S. Plante, S. Dubowsky, M. Hafez, D. Kacher, F. Jolesz, "Manipulation in MRI devices using electrostrictive polymer actuators: with an application to reconfigurable imaging coils," in
Proc. IEEE International Conference on Robotics and Automation ICRA '04, 3, 2498-2504 Vol.3 (2004).

[7] S. Rosset, M. Niklaus, P. Dubois, M., Dadras, H.R. Shea, "Mechanical properties of electroactive polymer microactuators with ion-implanted electrodes", in Electroactive Polymer Actuators and Devices (EAPAD) 2007, 6524, 652410-11, SPIE, San Diego, California, USA (2007).

[8] F. Carpi, P. Chiarelli, A. Mazzoldi, A., D. De Rossi, "Electromechanical characterisation of dielectric elastomer planar actuators: comparative evaluation of different electrode materials and different counterloads," Sensors and Actuators A: Physical 107(1), 85-95 (2003).

[9] M. Aschwanden and A. Stemmer, in Electroactive Polymer Actuators and Devices (EAPAD) 2007, vol 6524 (San Diego, California, USA: SPIE) pp $65241 \mathrm{~N}-10$.

[10] R. Pelrine, R. Kornbluh, J. Joseph, R. Heydt, Q. Pei, S. Chiba, "High-field deformation of elastomeric dielectrics for actuators," Materials Science and Engineering C 11(2), 89-100 (2000).

[11] A. Pimpin, Y. Suzuki, N. Kasagi, "Micro Electrostrictive Actuator with Metal Compliant Electordes for Flow Control Applications", Journal of Microelectromechanical Systems 16 753-764, 2007.

[12] P. Dubois, S. Rosset, S. Koster, J. Stauffer, S. Mikhailov, M. Dadras, N.-F. de Rooij, and H. Shea, "Microactuators based on ion implanted dielectric electroactive polymer (EAP) membranes", Sensors and Actuators A: Physical, vol. 130-131, pp. 147-154, 2006.

[13] S. Rosset, M. Niklaus, P. Dubois, H. R. Shea, "Mechanical characterization of a dielectric elastomer microactuator with ion-implanted electrodes", Sensors and Actuators A: Physical 144 (1) (2008) 185-193.

[14] S. Rosset, M. Niklaus, P. Dubois, H. Shea, "Metal ion implantation for the Fabrication of Stretchable Electrodes on Elastomers", Advanced Functional Materials, 19, 470-478, 2009.

[15] M. Niklaus, S. Rosset, M. Dadras, P. Dubois, H. Shea, "Microstructure of $5 \mathrm{keV}$ Gold implanted Polydimethylsiloxane", Scripta Materialia 59, pp. 893-896, 2008.

[16] P. Dubois, S. Rosset, M. Niklaus, M. Dadras, H. Shea, "Voltage control of the resonance frequency of a dielectric electroactive polymer (DEAP) membrane", IEEE/ASME Journal of Microelectromechanical systems, vol 17, pp. $1072-1081,2008$.

\section{CONTACT}

* Herbert Shea, herbert.shea@epfl.ch http://lmts.epfl.ch 